\title{
CANCER
}

\section{Tuning anticancer T cells}

Engineering T cells by transduction with antigen-specific $\mathrm{T}$ cell receptors (TCRs) or chimeric antigen receptors (CARs) that target a tumour-specific antigen can redirect a patient's own T cells to tumour cells. However, few tumours express truly specific antigens, and off-target activity of such redirected T cells can damage healthy tissues. Now, reporting in Nature Biotechnology, the group of Michel Sadelain presents a new approach: by engineering $\mathrm{T}$ cells to kill in response to a particular combination of two antigens, tumour specificity can be increased and "on-target,

off-tumour" side effects reduced.

The dual mode of $\mathrm{T}$ cell activation was achieved by transducing $\mathrm{T}$ cells with a TCR or CAR that provides suboptimal stimulation and requires functional complementation by a chimeric co-stimulatory receptor (CCR) that recognizes a second antigen. Initial experiments were carried out with $\mathrm{T}$ cells expressing a CAR directed at the $\mathrm{B}$ cell antigen CD19 and a CCR directed at prostate-specific membrane antigen (PSMA). The CAR was constructed from a CD19-specific single-chain variable fragment $(\mathrm{scFv})$ fused to the TCR $\zeta$-intracellular signalling domain (19z1), and the CCR consisted of a PSMA-specific scFv fused to the intracellular signalling domains of the co-stimulatory receptors $\mathrm{CD} 28$ and 4-1BB (P28BB).

Immunocompromised mice (non-obese diabetic/severe combined immunodeficient (NOD.SCID) $\mathrm{Il} 2 \mathrm{rg}^{-/-}$mice) were injected with a human $\mathrm{CD}_{19}{ }^{+} \mathrm{PSMA}^{+}$tumour cell line and treated with primary human peripheral blood $T$ cells expressing either 19z1, 19z1+P28BB or P28BB alone. Treatment with P28BB T cells had no effect on tumour growth, whereas treatment with CD19z1 $\mathrm{T}$ cells reduced the tumour burden, and treatment with $19 \mathrm{z} 1+\mathrm{P} 28 \mathrm{BB}$ $\mathrm{T}$ cells appeared to completely eradicate the tumour cells.

However, it was found that 19z1+P28BB T cells also showed activity against tumour cells expressing CD19 alone (thereby modelling potentially healthy tissue), so the approach was optimized to restrict $\mathrm{T}$ cell activity to tissues co-expressing both antigens. To this end, CARs with diminished activity were constructed, and the targeting of a combination of two prostate antigens, PSMA and prostate stem cell antigen (PSCA), was explored. Both antigens are promising targets for the treatment of metastatic prostate cancer, but neither is absolutely specific to prostate tissue. Experiments with different PSCA-specific CAR constructs in combination with $\mathrm{P} 28 \mathrm{BB}$ resulted in the development of engineered $\mathrm{T}$ cells that were strictly specific for tumour cells bearing both PSMA and PSCA, permanently eradicating these in experiments with immunocompromised mice as described above, while having no effect on cells expressing either PSMA or PSCA alone.

These results demonstrate that the selectivity and activity of engineered $\mathrm{T}$ cells can be restricted in a manner that reconciles potency with safety. Importantly, the authors point out

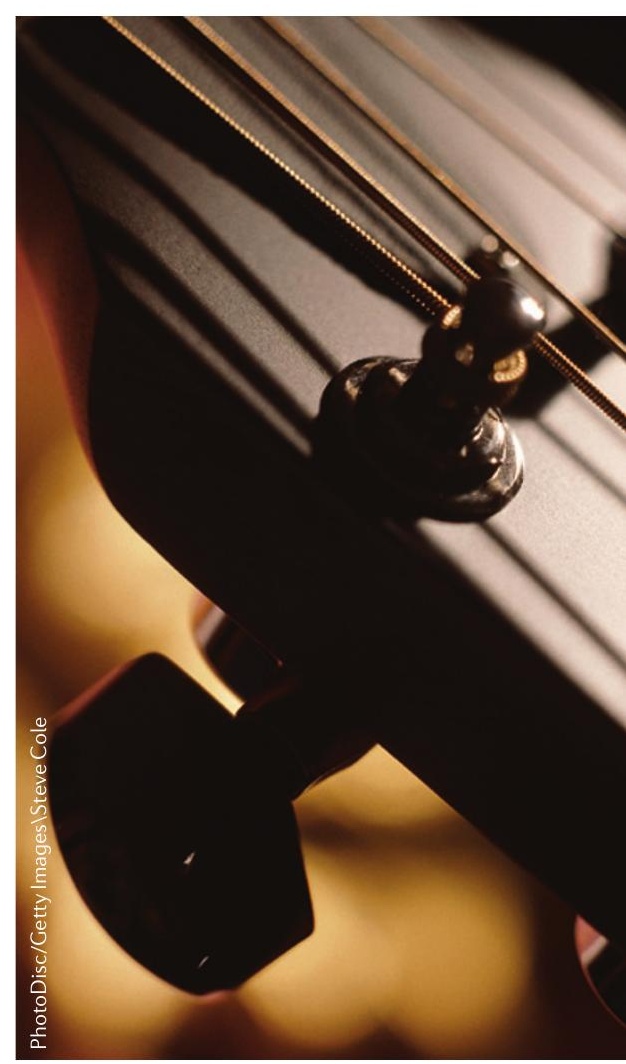

that by harnessing combinatorial antigen recognition, tumour-specific $T$ cells can be engineered even in the absence of a truly tumour-specific target - thus expanding the range of tumours that can potentially be treated by adoptive transfer of engineered T cells.

Alexandra Flemming

ORIGINAL RESEARCH PAPER Kloss, C. C. et al. Combinatorial antigen recognition with balanced signaling promotes selective tumour eradication by engineered T cells. Nature Biotech. 31, 71-75 (2013) 